\title{
Increased Resting Energy Expenditure and Insulin Resistance in Male Patients With Moderate-to Severe Obstructive Sleep Apnoea
}

\author{
M. BRÚSIK ${ }^{1}$, Z. ŠTRBOVÁ ${ }^{1}$, D. PETRÁŠOVÁ ${ }^{2}$, P. POBEHA $^{1}$, Z. KUKLIŠOVÁ $^{1}$, \\ R. TKÁČOVÁ ${ }^{1}$, P. JOPPA ${ }^{1}$
}

${ }^{1}$ Department of Respiratory Medicine, Faculty of Medicine, P. J. Šafárik University and L. Pasteur University Hospital, Košice, Slovak Republic, ${ }^{2}$ Laboratory of Research Biomodels, Faculty of Medicine, P. J. Šafárik University, Košice, Slovak Republic

Received December 16, 2015

Accepted May 13, 2016

On-line August 19, 2016

\section{Summary}

Obstructive sleep apnoea (OSA) has been associated with disturbances in energy metabolism and insulin resistance, nevertheless, the links between OSA severity, resting energy expenditure (REE) and insulin resistance (homeostasis model assessment, HOMA-IR) remained unexplored. Therefore, we investigated the effects of OSA severity on REE, and relationships between REE and HOMA-IR in patients with OSA. Forty men [mean (SD) age 49.4 (11.4) years] underwent overnight polysomnography; REE was assessed using indirect calorimetry. REE adjusted for fat-free mass (FFM) was higher in patients with moderate-to severe OSA [ $n=24$; body mass index (BMI) 31.1 (2.7) kg.m ${ }^{-2}$; apnoea-hypopnoea index (AHI) $\geq 15$ episodes. $\mathrm{h}^{-1}$ ] compared to participants with no clinically significant OSA $\left(n=16 ;\right.$ BMI 30.3 (2.2) kg.m ${ }^{-2} ;$ AHI $<15$ episodes. $\left.\mathrm{h}^{-1}\right)$ [median (interquartile range) 30.4 (26.1-31.3) versus 25.8 (24.6-27.3) kcal. $\left.\left.\mathrm{kg}^{-1} .24 \mathrm{~h}^{-1}, \mathrm{p}=0.005\right)\right]$. AHI and oxygen desaturation index (ODI) were directly related to REE/FFM ( $p=0.001 ; p<0.001$, respectively) and to HOMA-IR ( $p<0.001$ for both). In stepwise multiple linear models, REE/FFM was independently predicted by ODI $(\mathrm{p}<0.001)$ and age $(\mathrm{p}=0.028)\left(R^{2}=0.346\right) ;$ HOMA-IR was independently predicted by ODI only $\left(\mathrm{p}<0.001, R^{2}=0.457\right)$. In conclusion, male patients with moderate-to severe OSA have increased REE paralleled by impaired insulin sensitivity. Severity of nocturnal intermittent hypoxia reflected by ODI is an independent predictor of REE/FFM and HOMA-IR.

\section{Key words}

Obstructive sleep apnoea • Resting energy expenditure • Obesity

- Insulin resistance

\section{Corresponding author}

P. Joppa, Department of Respiratory Medicine and Tuberculosis, Faculty of Medicine, P. J. Šafárik University in Košice and L. Pasteur University Hospital, Rastislavova 43, Košice, Slovakia. Fax: (421) 55 615-2664. E-mail: pavol.joppa@upjs.sk

\section{Introduction}

Obstructive sleep apnoea (OSA) is characterized by repeated episodes of upper airway occlusion during sleep that are associated with hypoxia and arousals from sleep. Acutely, repetitive apnoeas and hypopnoeas during sleep trigger surges in sympathetic nervous system activity, blood pressure and heart rate (Dewan et al. 2015). Chronically, patients with OSA are at increased risk for insulin resistance (Ip et al. 2002, Polotsky et al. 2009, Kent et al. 2014), and have an increased cardiovascular morbidity and mortality (Kasai et al. 2012).

In the frame of The European Sleep Apnoea Database (ESADA) study we have recently reported, in a cohort of 5103 subjects, extraordinarily high prevalence of obesity among patients with OSA: morbid obesity [body mass index $(\mathrm{BMI}) \geq 35 \mathrm{~kg} \cdot \mathrm{m}^{-2}$ ] was present in $21.1 \%$ of males and $28.6 \%$ of females (Hedner et al. 2011). The global obesity epidemic, and the high prevalence of obesity in OSA has stimulated intense interest in the study of homeostatic mechanisms governing the energy balance in these subjects. Previous reports on energy expenditure in patients with OSA are 
scarce, and yielded to inconsistent results (Ryan et al. 1995, Stenlof et al. 1996, Lin et al. 2002, Major et al. 2007, Kezirian et al. 2008, Ucok et al. 2011, de Jonge et al. 2012). Methodological discrepancies largely contributed to these inconsistencies: whereas the former studies mostly did not, the more recent analyses have adjusted resting energy expenditure (REE) for fat-free mass (FFM). Interestingly, three studies demonstrated increases in REE in patients with OSA compared to healthy subjects after adjustments for FFM (Stenlof et al. 1996, Ucok et al. 2011, Fekete et al. 2015).

Mechanisms affecting REE in patients with OSA are not well understood. In a recent study that evaluated a range of factors influencing REE in obese Caucasian women, a history of OSA, and impaired glycemic status were both significant predictors of REE (Hagedorn et al. 2012). Nevertheless, no diagnostic polysomnography was performed in that study, and thus relationships between the severity of OSA and REE remained unclear. In addition, insulin resistance was not objectively assessed either by direct (euglycemic hyperinsulinemic clamp) nor by indirect (homeostasis model assessment, HOMA-IR) methods in any of the previous studies on REE in patients with OSA. Nevertheless, increases in REE were related to decreases in insulin sensitivity in healthy subjects following voluntary sleep restriction (Bosy-Westphal et al. 2008). Therefore, the aim of the present study was to analyze relationships between OSA severity, insulin sensitivity and REE in obese patients with OSA diagnosed by overnight polysomnography.

\section{Patients and Methods}

\section{Subjects}

Clinically stable men with suspected OSA referred to the sleep unit at a tertiary referral teaching hospital (Department of Respiratory Medicine, Faculty of Medicine, P. J. Safarik University and L. Pasteur University Hospital, Kosice, Slovakia) for a diagnostic sleep study were prospectively recruited. Exclusion criteria were as follows: 1) chronic respiratory diseases other than OSA, such as bronchial asthma, chronic obstructive pulmonary disease, restrictive pulmonary disorders or hypoventilation syndrome; 2) known metabolic, endocrine, autoimmune, hepatic or renal disorders; 3) malignancy; 4) overt heart failure; or 5) regular use of sedatives, antidepressant or antipsychotic medication or alcohol. In all subjects, pulmonary function tests were assessed with the use of bodyplethysmography (Ganshorn, Germany) in accordance with European Respiratory Society standards (Miller et al. 2005). The study was conducted in accordance with the principles of Helsinki declaration, approved by the L. Pasteur University Hospital ethics committee, and all subjects provided written informed consent.

\section{Sleep assessments}

All participants underwent full attended diagnostic overnight polysomnography (Alice 4; Respironics Inc., Murrysville, Pennsylvania, USA), comprising continuous recording of electroencephalography (EEG), electrooculography, electromyography, electrocardiography, thoracic and abdominal impedance belts, thermistor for nasal and oral airflow, pulse oximetry and microphone for snoring. All records were scored manually following the American Academy for Sleep Medicine (AASM) 2007 guidelines (Berry et al. 2012). Apnoea was identified as a drop in airflow of $>90 \%$ from the baseline excursion for $\geq 10 \mathrm{~s}$; hypopnoea was defined as a reduction in airflow of $\geq 50 \%$ of baseline for $\geq 10 \mathrm{~s}$ accompanied either by a decrease in hemoglobin saturation for $\geq 3 \%$, an EEG-recorded arousal, or both. The apnoea/hypopnoea index (AHI) was defined as the number of apnoea and hypopnoea episodes per hour of sleep. Oxygen desaturation index (ODI) was defined as the number of oxygen desaturations of hemoglobin of $\geq 3 \%$ per hour of sleep. The classification of OSA severity was based on the AASM guidelines as follows (Berry et al. 2012): mild: $\mathrm{AHI} \geq 5$ and $<15$ episodes. $\mathrm{h}^{-1}$; moderate: $\mathrm{AHI} \geq 15$ and $<30$ episodes. $\mathrm{h}^{-1}$; and severe: AHI $\geq 30$ episodes. $\mathrm{h}^{-1}$. The entire cohort was divided based on OSA severity into two groups: 1) patients with moderate-to severe OSA $\left(\mathrm{AHI} \geq 15\right.$ episodes. $\mathrm{h}^{-1}$ ), that was shown to be associated with important clinical consequences (such as increased cardiovascular risk) irrespective on the presence of symptoms (Epstein et al. 2009), and 2) participants with no clinically significant $\mathrm{OSA}\left(\mathrm{AHI}<15\right.$ episodes. $\left.\mathrm{h}^{-1}\right)$.

\section{Body composition}

Bioelectrical impedance analysis (BIA) (Bodystat 1500, Bodystat, Isle of Man, UK) was performed after overnight fasting to assess the amount of fat mass and FFM (in $\mathrm{kg}$ ). Impedance was measured between the right wrist and right ankle using a tetrapolar electrode system and the obtained data were analyzed using the manufacturer's software. To adjust for 
differences in body surface, both fat mass and FFM were divided by squared height. Subsequently, the fat-free mass index [FFMI; fat-free mass in kg.(height in $\mathrm{m})^{-2}$ ] was used as a surrogate measure of skeletal muscle mass, while the fat mass index [FMI; fat mass in kg.(height in $\mathrm{m}^{-2}$ ] was used as a measure for body fat mass.

\section{Biochemical analyses}

In all patients, peripheral venous blood samples from the antecubital vein were collected between 7.00 and 8.00 a.m. after overnight fasting. Routine biochemical and hematological assessments were performed at the day of collection. Serum insulin was determined with electrochemiluminiscence immunoassay kits (Elecsys) on Roche Elecsys 1010/2010 and modular analytics E170 immunoassay analyzers (Roche Diagnostics GmbH, Germany); plasma glucose was measured by the glucose oxidase method on a Beckman autoanalyzer. Insulin resistance was estimated by the HOMA-IR using the following formula: fasting serum insulin $\left(\mathrm{mU} . \mathrm{l}^{-1}\right) \mathrm{x}$ fasting plasma glucose $\left(\mathrm{mmol} . \mathrm{1}^{-1}\right) / 22.5$ (Matthews et al. 1985).

Serum leptin, soluble leptin receptor (sOB-R) and adiponectin were determined by the enzyme-linked immunosorbent assays (DRG, Germany; BioVendor, Germany). Leptin to sOB-R ratio was used to calculate the free-leptin index (FLI).

\section{Measurements of resting energy expenditure}

Resting energy expenditure (REE) was measured by open-circuit indirect calorimetry using the Ergostik system (Geratherm, Germany). Patients were breathing through an oro-nasal interface that enabled a breath-tobreath analysis of gases. Internal pacing frequency of the gas analyzer was $125 \mathrm{~Hz}$. Oxygen concentration was measured by an electrochemical sensor and carbon dioxide concentration by infrared absorption method. The system was calibrated daily. Prior to each metabolic test, the flowmeter was calibrated using a 3 liter calibration syringe (Pulmonary Data Services Inc., USA) and the gas analyzers were calibrated using a two-point calibration method with certified gases $\left(15.9 \% \mathrm{O}_{2}, 5.0 \% \mathrm{CO}_{2}\right.$, Linde Gas, Slovakia). Patients were examined after at least $10 \mathrm{~h}$ fast, and refraining from smoking, alcohol and caffeine consumption, and vigorous physical activity for $24 \mathrm{~h}$ prior to the test. Patients were placed in a comfortable reclined position, allowed to rest at least 30 min prior to measurement. The measurements were recorded within a 30 min period between 8.00 and 9.00 a.m., and REE was calculated from oxygen consumption and carbon dioxide production using the abbreviated Weir equation (Weir 1949). Since the absolute values of REE depend on body size, we calculated the ratio of REE to fat-free mass (REE/FFM) as suggested previously (Rotella and Dicembrini 2015).

\section{Statistical analyses}

Statistical analyses were performed using SPSS software version 14.0 (SPSS Inc., USA). The Kolmogorov-Smirnov test of normality was applied. Differences between groups in normally distributed variables were tested by unpaired $t$-test and in nonnormally distributed variables by Wilcoxon-MannWhitney $U$-test. Chi-square test was used to compare the proportion of ever smokers between groups.

Least-squares linear regression analysis was used to assess the unadjusted relationships between the REE/FFM or HOMA-IR and independent variables. Pearson product-moment correlation coefficient $(R)$ is reported to show the degree of linear relationship between variables. In the multivariate analyses, multiple linear regression models were used with REE/FFM or HOMA-IR as the dependent variable, and age, AHI, ODI, arousal index, and FMI as independent variables. Coefficient of determination $\left(R^{2}\right)$ is reported to indicate the model fit, and percentage of variance of the dependent variables explained by the predictors included in the model.

A $p$ value of $<0.05$ was considered statistically significant. Continuous variables with normal distribution are shown as means (SD), non-normally distributed variables as median (interquartile range).

\section{Results}

\section{Study population}

Forty men with suspected OSA [mean age, 49.4 (11.4) years] with an overweight to obese BMI [mean, $30.8(2.6) \mathrm{kg} \cdot \mathrm{m}^{-2}$ ] were recruited. OSA was diagnosed in 36 patients (mild in 12, moderate in 5 and severe in 19 patients, respectively), whereas four men had no sleep-disordered breathing. Participants were divided into two groups according to the AHI: the first was formed by 16 men with no clinically significant OSA $\left(\mathrm{AHI}<15\right.$ episodes. $\left.\mathrm{h}^{-1}\right)$, and the second by 24 male patients with the diagnosis of moderate-to severe OSA $\left(\mathrm{AHI} \geq 15\right.$ episodes. $\left.\mathrm{h}^{-1}\right)$. 
Table 1. Demographic, anthropometric, lung function and polysomnography parameters.

\begin{tabular}{|c|c|c|c|}
\hline & AHI $<15$ episodes. ${ }^{-1}$ & AHI $\geq 15$ episodes. $^{-1}$ & $\mathbf{p}$ \\
\hline Participants, number & 16 & 24 & \\
\hline Age, years & $47.8(13.3)$ & $50.4(10.0)$ & 0.669 \\
\hline Ever smokers, \% & 25.0 & 37.5 & 0.630 \\
\hline$B M I, k g \cdot m^{-2}$ & $30.3(2.2)$ & $31.1(2.7)$ & 0.336 \\
\hline$F F M I, k g \cdot m^{-2}$ & $21.9(1.2)$ & $21.8(1.4)$ & 0.647 \\
\hline$F M I, k g . m^{-2}$ & $8.3(1.4)$ & $9.3(1.8)$ & 0.067 \\
\hline$F V C, \%$ predicted & $103.7(8.5)$ & $92.5(12.6)$ & 0.003 \\
\hline$F E V_{1}, \%$ predicted & $99.5(8.4)$ & $90.7(11.7)$ & 0.014 \\
\hline AHI, episodes. $h^{-1}$ & $9.5(4.9-12.1)$ & $49.0(31.2-67.5)$ & $<0.001$ \\
\hline ODI, episodes. $h^{-1}$ & $4.2(2.8-8.1)$ & $39.1(23.1-64.1)$ & $<0.001$ \\
\hline Arousal index, episodes. $h^{-1}$ & $15.1(13.0-19.0)$ & $56.7(38.3-73.7)$ & $<0.001$ \\
\hline ESS, points & $6.0(4.5-7.5)$ & $7.0(4.5-9.0)$ & 0.489 \\
\hline
\end{tabular}

Data are presented as mean (SD) or median (interquartile range) as appropriate, unless indicated otherwise. AHI - apnoea/hypopnoea index, BMI - body mass index, FFMI - fat-free mass index, FMI - fat mass index, FVC - forced vital capacity, FEV ${ }_{1}$ - forced expiratory volume in $1 \mathrm{~s}$, ODI - oxygen desaturation index, ESS - Epworth Sleepiness Scale score.

Table 2. Glucose metabolism parameters and serum adipokine levels.

\begin{tabular}{|c|c|c|c|}
\hline & AHI $<15$ episodes. $h^{-1}$ & $A H I \geq 15$ episodes. $h^{-1}$ & $\mathbf{p}$ \\
\hline Glucose, mmol.l $l^{-1}$ & $5.1(4.6-5.3)$ & $5.3(4.7-5.7)$ & 0.143 \\
\hline Insulin, $m U . l^{-1}$ & $8.2(5.9-11.8)$ & $11.6(8.1-13.8)$ & 0.054 \\
\hline$H O M A-I R$ & $1.7(1.4-2.2)$ & $2.6(2.1-3.6)$ & 0.010 \\
\hline Adiponectin, $\mu g . m l^{-1}$ & $5.0(2.8-8.4)$ & $6.1(4.3-8.8)$ & 0.230 \\
\hline Leptin, $n g . m l^{-1}$ & $16.3(12.0-24.0)$ & $19.2(14.7-25.6)$ & 0.383 \\
\hline$s O B-R, n g \cdot m l^{-1}$ & $28.8(26.2-48.3)$ & $37.1(25.4-46.4)$ & 0.558 \\
\hline$F L I$ & $0.5(0.3-0.9)$ & $0.5(0.4-0.8)$ & 0.858 \\
\hline
\end{tabular}

Data are presented as median (interquartile range). AHI - apnoea/hypopnoea index, HOMA-IR - homeostasis model-assessed insulin resistance, sOB-R - soluble leptin receptor, FLI - free leptin-index (leptin to SOB-R ratio).

Table 1 displays demographic data, lung function and body composition parameters in the two groups. No significant differences were observed between the groups in the mean age, BMI, FMI and FFMI. Although no restrictive lung disease or obstructive airflow limitation was present in neither of the two groups, patients with moderate-to severe OSA had significantly lower lung volumes than individuals with no clinically significant OSA (Table 1).

Glucose metabolism parameters and adipokine concentrations in the two groups are displayed in Table 2. Although subjects in both groups were euglycemic, those with moderate-to severe OSA had higher HOMA-IR ( $p=0.01)$. No differences were seen in plasma concentrations of adipokines between the two
BMI-matched groups.

Resting energy expenditure and insulin resistance

Basal metabolic rate expressed as REE/FFM was significantly higher in patients with moderate-to severe OSA compared to participants with no clinically significant OSA [30.4 (26.1-31.3) versus 25.8 (24.6-27.3) kcal.kg ${ }^{-1} .24 \mathrm{~h}^{-1}, \mathrm{p}=0.005$, Fig. 1]. Parameters reflecting OSA severity, i.e. AHI, ODI and arousal index were directly related to $\mathrm{REE} / \mathrm{FFM} \quad(\mathrm{p}=0.001 ; \mathrm{p}<0.001$; $\mathrm{p}=0.004$, respectively) and to HOMA-IR ( $<<0.001$ for all). A significant positive linear relationship was present between REE/FFM and HOMA-IR ( $<<0.05)$ (Table 3).

In the stepwise multiple linear regression models with age, AHI, ODI, arousal index and FMI as 
co-variates, age $(p=0.028)$ and ODI $(p<0.001)$ were independent predictors of REE/FFM $\left(R^{2}=0.346\right)$. HOMA-IR was independently predicted by ODI only $\left(\mathrm{p}<0.001, R^{2}=0.457\right)$.

Table 3. Bivariate regression analyses with REE/FFM and HOMA-IR as dependent variables.

\begin{tabular}{lcccc}
\hline \multirow{2}{*}{$\begin{array}{l}\text { Independent } \\
\text { variable }\end{array}$} & \multicolumn{2}{c}{ REE/FFM } & \multicolumn{2}{c}{ HOMA-IR } \\
\cline { 2 - 5 } Age & $\mathbf{R}$ & $\mathbf{p}$ & $\mathbf{R}$ & $\mathbf{p}$ \\
\hline FMI & -0.254 & 0.114 & -0.087 & 0.600 \\
AHI & 0.245 & 0.127 & 0.376 & 0.018 \\
ODI & 0.500 & 0.001 & 0.676 & $<0.001$ \\
Arousal index & 0.442 & 0.004 & 0.632 & $<0.001$ \\
HOMA-IR & 0.320 & 0.047 & - & - \\
\hline
\end{tabular}

REE/FFM - resting energy expenditure adjusted for fat-free mass, HOMA-IR - homeostasis model-assessed insulin resistance, FMI - fat mass index, AHI - apnoea/hypopnoea index, ODI oxygen desturation index.

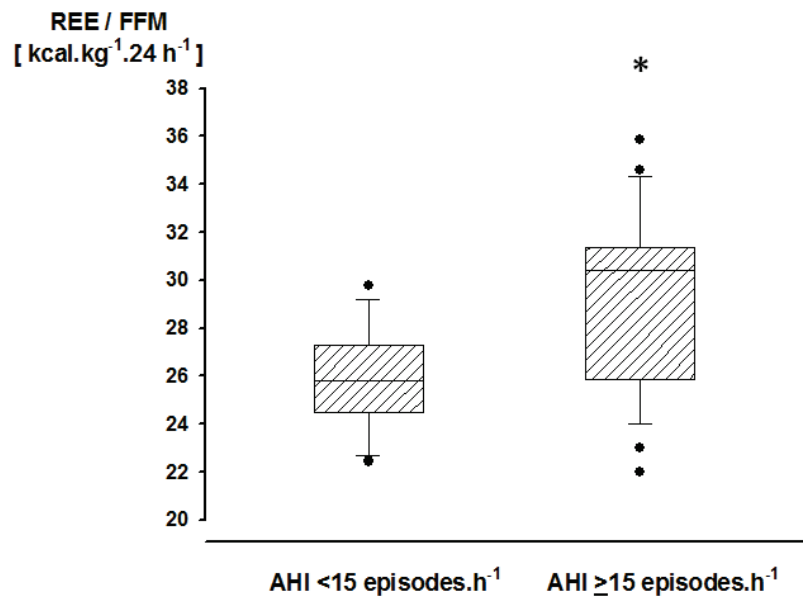

Fig. 1. Resting energy expenditure in male subjects without and with moderate-to severe obstructive sleep apnoea. $* p<0.01$ compared to subjects with $\mathrm{AHI}<15$ episodes. $\mathrm{h}^{-1}$, REE/FFM resting energy expenditure adjusted for fat-free mass, AHI apnoea/hypopnoea index.

\section{Discussion}

A worldwide epidemic of obesity, a critical risk factor for diabetes and cardiovascular diseases, has led to intense studies into mechanisms that govern energy balance and insulin resistance in obesity and associated disorders, including sleep-disordered breathing. The present study demonstrates that male patients with moderate-to severe OSA have increased REE, paralleled by impaired insulin sensitivity compared to BMI-matched men with no clinically significant OSA. By concomitant assessment of REE, body composition and insulin sensitivity in a well-defined cohort of middle-aged men assessed by overnight polysomnography, our findings demonstrate that OSA severity as reflected by oxygen desaturation index is an independent predictor of both, basal metabolic rate and HOMA-IR in such patients.

Increased body size-corrected energy expenditure in patients with OSA compared to control subjects was previously demonstrated in some (Stenlof $e t$ al. 1996, Lin et al. 2002, Ucok et al. 2011) but not all studies (Ryan et al. 1995, Major et al. 2007). In addition, while some studies demonstrated a positive association between the indices of OSA severity and REE (Kezirian et al. 2008, de Jonge et al. 2012), the study of Major et al. (2007) failed to identify such relationship. A recent study, that included by far the largest number of overweight-to obese male patients with OSA, reported increased REE corrected for lean body mass in comparison with control subjects without OSA (Fekete et al. 2015). Importantly, the present findings extend previous observations of increased REE among patients with OSA further: here we demonstrate for the first time that insulin resistance assessed by HOMA-IR was related to REE in OSA. This observation parallels previous observations in healthy individuals by Bosy-Westphal et al. (2008) who reported decreases in insulin sensitivity or a higher insulin secretion in those healthy individuals who developed increases in REE following voluntary sleep deprivation.

Several mechanisms might contribute to increases in basal metabolic rate in patients with OSA. Interestingly, animal studies suggested that sustained hypoxia per se reduces energy metabolism, in association with attenuated lipid uptake in most tissues (Jun et al. 2013). In addition, administration of hypoxia-inducible factor- $1 \alpha$ antisense oligonucleotides resulted in increased energy expenditure in mice (Shin et al. 2012). In contrast, however, activation of hypoxia-inducible factor- $1 \alpha$ by ascent to high altitude is considered one of potential basal metabolism increasing and weight-losing strategies among humans (Palmer and Clegg 2014). OSA is characterized by chronic intermittent hypoxia during sleep that has been consistently associated with sympathetic activation both in animal models of OSA and also in patients with OSA (Phillips et al. 2000, Grassi et al. 2005, Dewan et al. 2015, Mesarwi et al. 2015). In addition, higher daytime urinary norepinephrine was 
found in patients with OSA compared to healthy snorers and, importantly, plasma norepinephrine was positively correlated with energy expenditure during sleep in these patients (Stenlof et al. 1996). Importantly, apart from sympathetic activation resulting from chronic intermittent hypoxia, other factors likely contribute to increased energy expenditure in patients with OSA. Indeed, increases in energy expenditure resulting from augmented but ineffective respiratory effort of inspiratory muscles (Chien et al. 2010) may also contribute to the nocturnal increases of sympathetic nerve activity, and thus to increased metabolic rate in OSA patients. In the present study, REE/FFM was independently predicted by ODI which is an objective marker of OSA severity and of the degree of nocturnal intermittent hypoxia. Measurements of sympathetic nerve activity were beyond the scope of the present investigation. Therefore, further studies are needed to elucidate the effects of hypoxia-induced sympathetic activation on energy metabolism in patients with OSA in more detail.

Among other mechanisms affecting energy balance, leptin signaling has to be considered. Leptin, an adipocyte-derived hormone which circulates in the plasma at concentrations proportional to fat mass, crosses the blood brain barrier where it activates its receptors in the hypothalamus to control body weight by reducing food intake and increasing energy expenditure (Rosenbaum and Leibel 2014). Importantly, leptin signaling interacts with systemic hypoxia to increase sympathetic nerve activity and basal metabolic rate at high altitude exposure (Enriori et al. 2011, Hansen and Sander 2003). In the present study we took great care to match patients with moderate-to severe OSA to participants with no clinically significant OSA for BMI, and neither FMI nor any of the adipokine level (leptin, adiponectin, sOB-R, FLI) differed between the two groups.

In our recent studies within the large ESADA cohort of 5294 participants we have demonstrated that OSA severity independently predicted glycemic health in non-diabetic subjects on one hand, and increased the risk of concomitant type 2 diabetes and worse diabetic control in diabetics on the other (Kent et al. 2014). Insulin sensitivity was not assessed within these large epidemiological cohorts, and thus our present results complement previous findings by demonstrating a close relationship between HOMA-IR and indices of OSA severity such as AHI, arousal index and ODI. In the present study ODI was the only independent predictor of HOMA-IR in multivariate analyses. In animal studies, chronic intermittent hypoxia induced insulin resistance in both obese and lean mice (Polotsky et al. 2003, Iiyori et al. 2007, Drager et al. 2011) and its reversal by hypoxia-inducible factor inhibitor was followed by an improvement of insulin sensitivity (Shin et al. 2012).

There are several limitations to this study. First, only a limited number of patients were studied. However, compared to participants with no clinically significant OSA, patients with moderate-to severe OSA had median values of REE/FFM increased by $18 \%$, and HOMA-IR by $54 \%$. Therefore, although our results are robust to gain some understanding on the role of OSA severity in sleep-disordered breathing-related increases in metabolic rate, they should be considered preliminary and hypothesis generating. Second, exploring gender differences in the outcomes of interest was beyond the scope of the present study, and only men were recruited. Nevertheless, gender appears to represent a significant determinant of fuel metabolism and its response to various physiological and pathological stimuli (Henderson 2014). In the future, gender differences in basal metabolism response to OSA and its reversal by therapy have to be considered. Furthermore, insulin sensitivity was approximated by using HOMA-IR instead of the euglycemic insulin clamp. Nevertheless, insulin sensitivity indices derived from the homeostatic model are strongly related to values obtained by the clamp method (Wallace et al. 2004). Final limitation of the study relates to its cross-sectional nature that does not allow for the determination of time-course relationship between REE, HOMA-IR and the severity of OSA. Nevertheless, concomitant measurements, within one group of patients with sleep-disordered breathing, of REE, body composition, insulin sensitivity and of serum adipokines are unique and represent strength of the study.

In conclusion, our study highlights the role of sleep apnoea severity in the increases of energy expenditure in patients with moderate-to severe OSA that are paralleled by an impairment of insulin sensitivity. Our observation suggests that the reversal of OSA by the application of continuous positive airway pressure (CPAP) may potentially normalize the increased energy expenditure in patients with OSA. Indeed, a recent study that investigated the effect of CPAP treatment on REE demonstrated a normalization of initially increased nocturnal REE after six months of CPAP use in OSA patients compliant with the therapy (Bamberga et al. 2015). In line with this observation, a recent meta-analysis documented a small but significant weight gain after the 
initiation of CPAP therapy (Drager et al. 2015). Further studies are needed to address the pathological mechanisms underlying the observed relationships in more details.

\section{Acknowledgements}

This work was supported by the Slovak Research and Development Agency under the contract No. APVV0134-11, and by grants VEGA 1/0863/15 and VEGA 1/0208/16 of the Ministry of Education, Slovakia.

\section{Conflict of Interest}

There is no conflict of interest.

\section{References}

BAMBERGA M, RIZZI M, GADALETA F, GRECHI A, BAIARDINI R, FANFULLA F: Relationship between energy expenditure, physical activity and weight loss during CPAP treatment in obese OSA subjects. Respir Med 109: 540-545, 2015.

BERRY RB, BUDHIRAJA R, GOTTLIEB DJ, GOZAL D, IBER C, KAPUR VK, MARCUS CL, MEHRA R, PARTHASARATHY S, QUAN SF, REDLINE S, STROHL KP, DAVIDSON WARD SL, TANGREDI MM; AMERICAN ACADEMY OF SLEEP MEDICINE: Rules for scoring respiratory events in sleep: update of the 2007 AASM Manual for the Scoring of Sleep and Associated Events. J Clin Sleep Med 15: 597-619, 2012.

BOSY-WESTPHAL A, HINRICHS S, JAUCH-CHARA K, HITZE B, LATER W, WILMS B, SETTLER U, PETERS A, KIOSZ D, MUELLER MJ: Influence of partial sleep deprivation on energy balance and insulin sensitivity in healthy women. Obes Facts 1: 266-273, 2008.

CHIEN MY, WU YT, LEE PL, CHANG YJ, YANG PC: Inspiratory muscle dysfunction in patients with severe obstructive sleep apnoea. Eur Respir J 35: 373-380, 2010.

DE JONGE L, ZHAO X, MATTINGLY MS, ZUBER SM, PIAGGI P, CSAKO G, CIZZA G; NIDDK SLEEP EXTENSION STUDY GROUP: Poor sleep quality and sleep apnea are associated with higher resting energy expenditure in obese individuals with short sleep duration. J Clin Endocrinol Metab 97: 2881-2889, 2012.

DEWAN NA, NIETO FJ, SOMERS VK: Intermittent hypoxemia and OSA: implications for comorbidities. Chest 147: 266-274, 2015.

DRAGER LF, LI J, REINKE C, BEVANS-FONTI S, JUN JC, POLOTSKY VY: Intermittent hypoxia exacerbates metabolic effects of diet-induced obesity. Obesity (Silver Spring) 19: 2167-2174, 2011.

DRAGER LF, BRUNONI AR, JENNER R, LORENZI-FILHO G, BENSENOR IM, LOTUFO PA: Effects of CPAP on body weight in patients with obstructive sleep apnoea: a meta-analysis of randomised trials. Thorax 70: 258-264, 2015.

ENRIORI PJ, SINNAYAH P, SIMONDS SE, RUDAZ C, COWLEY MA: Leptin action in the dorsomedial hypothalamus increases sympathetic tone to brown adipose tissue in spite of systemic leptin resistance. J Neurosci 31: 12189-12197, 2011.

EPSTEIN LJ, KRISTO D, STROLLO PJ, FRIEDMAN N, MALHOTRA A, PATIL SP, RAMAR K, ROGERS R, SCHWAB RJ, WEAVER EM, WEINSTEIN MD: Clinical guideline for the evaluation, management and longterm care of obstructive sleep apnea in adults. J Clin Sleep Med 5: 263-276, 2009.

FEKETE K, BOUTOU AK, PITSIOU G, CHAVOUZIS N, PATAKA A, ATHANASIOU I, ILONIDIS G, KONTAKIOTIS T, ARGYROPOULOU P, KIOUMIS I: Resting energy expenditure in OSAS: the impact of a single CPAP application. Sleep Breath 20: 121-128, 2016.

GRASSI G, FACCHINI A, TREVANO FQ, DELL'ORO R, ARENARE F, TANA F, BOLLA G, MONZANI A, ROBUSCHI M, MANCIA G: Obstructive sleep apnea-dependent and -independent adrenergic activation in obesity. Hypertension 46: 321-325, 2005.

HAGEDORN T, POGGIOGALLE E, SAVINA C, COLETTI C, PAOLINI M, SCAVONE L, NERI B, DONINI LM: Indirect calorimetry in obese female subjects: factors influencing the resting metabolic rate. World J Exp Med 2: 58-64, 2012.

HANSEN J, SANDER M: Sympathetic neural overactivity in healthy humans after prolonged exposure to hypobaric hypoxia. J Physiol 546: 921-929, 2003. 
HEDNER J, GROTE L, BONSIGNORE M, MCNICHOLAS W, LAVIE P, PARATI G, SLIWINSKI P, BARBÉ F, DE BACKER W, ESCOURROU P, FIETZE I, KVAMME JA, LOMBARDI C, MARRONE O, MASA JF, MONTSERRAT JM, PENZEL T, PRETL M, RIHA R, RODENSTEIN D, SAARESRANTA T, SCHULZ R, TKACOVA R, VARONECKAS G, VITOLS A, VRINTS H, ZIELINSKI J: The European Sleep Apnoea Database (ESADA): report from 22 European sleep laboratories. Eur Respir J 38: 635-642, 2011.

HENDERSON GC: Sexual dimorphism in the effects of exercise on metabolism of lipids to support resting metabolism. Front Endocrinol (Lausanne) 5: 162, 2014.

IIYORI N, ALONSO LC, LI J, SANDERS MH, GARCIA-OCANA A, O'DOHERTY RM, POLOTSKY VY, O'DONNELL CP: Intermittent hypoxia causes insulin resistance in lean mice independent of autonomic activity. Am J Respir Crit Care Med 175: 851-857, 2007.

IP MS, LAM B, NG MM, LAM WK, TSANG KW, LAM KS: Obstructive sleep apnea is independently associated with insulin resistance. Am J Respir Crit Care Med 165: 670-676, 2002.

JUN JC, SHIN MK, YAO Q, DEVERA R, FONTI-BEVANS S, POLOTSKY VY: Thermoneutrality modifies the impact of hypoxia on lipid metabolism. Am J Physiol Endocrinol Metab 304: E424-E435, 2013.

KASAI T, FLORAS JS, BRADLEY TD: Sleep apnea and cardiovascular disease: a bidirectional relationship. Circulation 126: 1495-1510, 2012.

KENT BD, GROTE L, BONSIGNORE MR, SAARESRANTA T, VERBRAECKEN J, LÉVY P, SLIWINSKI P, TKACOVA R, KVAMME JA, FIETZE I, HEDNER J, MCNICHOLAS WT; EUROPEAN SLEEP APNOEA DATABASE COLLABORATORS: Sleep apnoea severity independently predicts glycaemic health in nondiabetic subjects: the ESADA study. Eur Respir J 44: 130-139, 2014.

KEZIRIAN EJ, KIRISOGLU CE, RILEY RW, CHANG E, GUILLEMINAULT C, POWELL NB: Resting energy expenditure in adults with sleep disordered breathing. Arch Otolaryngol Head Neck Surg 134: 1270-1275, 2008.

LIN CC, CHANG KC, LEE KS: Effects of treatment by laser-assisted uvuloplasty on sleep energy expenditure in obstructive sleep apnea patients. Metabolism 51: 622-627, 2002.

MAJOR GC, SERIES F, TREMBLAY A: Does the energy expenditure status in obstructive sleep apnea favour a positive energy balance? Clin Invest Med 30: E262-E268, 2007.

MATTHEWS DR, HOSKER JP, RUDENSKI AS, NAYLOR BA, TREACHER DF, TURNER RC: Homeostasis model assessment: insulin resistance and beta-cell function from fasting plasma glucose and insulin concentrations in man. Diabetologia 28: 412-419, 1985.

MESARWI OA, SHARMA EV, JUN JC, POLOTSKY VY: Metabolic dysfunction in obstructive sleep apnea: a critical examination of underlying mechanisms. Sleep Biol Rhythms 13: 2-17, 2015.

MILLER MR, HANKINSON J, BRUSASCO V, BURGOS F, CASABURI R, COATES A, CRAPO R, ENRIGHT P, VAN DER GRINTEN CP, GUSTAFSSON P, JENSEN R, JOHNSON DC, MACINTYRE N, MCKAY R, NAVAJAS D, PEDERSEN OF, PELLEGRINO R, VIEGI G, WANGER J; ATS/ERS TASK FORCE: Standardisation of spirometry. Eur Respir J 26: 319-338, 2005.

PALMER BF, CLEGG DJ: Ascent to altitude as a weight loss method: the good and bad of hypoxia inducible factor activation. Obesity (Silver Spring) 22: 311-317, 2014.

PHILLIPS BG, KATO M, NARKIEWICZ K, CHOE I, SOMERS VK: Increases in leptin levels, sympathetic drive, and weight gain in obstructive sleep apnea. Am J Physiol Heart Circ Physiol 279: H234-H237, 2000.

POLOTSKY VY, LI J, PUNJABI NM, RUBIN AE, SMITH PL, SCHWARTZ AR, O'DONNELL CP: Intermittent hypoxia increases insulin resistance in genetically obese mice. J Physiol 552: 253-264, 2003.

POLOTSKY VY, PATIL SP, SAVRANSKY V, LAFFAN A, FONTI S, FRAME LA, STEELE KE, SCHWEIZTER MA, CLARK JM, TORBENSON MS, SCHWARTZ AR: Obstructive sleep apnea, insulin resistance, and steatohepatitis in severe obesity. Am J Respir Crit Care Med 179: 228-234, 2009.

ROSENBAUM M, LEIBEL RL: 20 years of leptin: role of leptin in energy homeostasis in humans. $J$ Endocrinol 223: T83-T96, 2014.

ROTELLA CM, DICEMBRINI I: Measurement of body composition as a surrogate evaluation of energy balance in obese patients. World J Methodol 5: 1-9, 2015.

RYAN CF, LOVE LL, BUCKLEY PA: Energy expenditure in obstructive sleep apnea. Sleep 18: 180-187, 1995. 
SHIN MK, DRAGER LF, YAO Q, BEVANS-FONTI S, YOO DY, JUN JC, AJA S, BHANOT S, POLOTSKY VY: Metabolic consequences of high-fat diet are attenuated by suppression of HIF-1 $\alpha$. PLoS One 7: e46562, 2012.

STENLOF K, GRUNSTEIN R, HEDNER J, SJOSTROM L: Energy expenditure in obstructive sleep apnea: effects of treatment with continuous positive airway pressure. Am J Physiol Endocrinol Metab 34: E1036-E1043, 1996.

UCOK K, AYCICEK A, SEZER M, FIDAN F, AKGUN L, AKKAYA M, UNLU M: Resting metabolic rate and anthropometric measurements in male sleep apnea patients. Intern Med 50: 833-838, 2011.

WALLACE TM, LEVY JC, MATTHEWS DR: Use and abuse of HOMA modeling. Diabetes Care 27: 1487-1495, 2004.

WEIR JB: New methods for calculating metabolic rate with special reference to protein metabolism. J Physiol 109: 1-9, 1949. 